\title{
Individualised computer generated nutrition information plus iterative feedback reduced dietary fat and increased fruit and vegetable intake
}

\author{
Brug J, Glanz K, Van Assema P, et al. The impact of computer-tailored feedback and iterative feedback on fat, fruit, and vegetable \\ intake. Health Educ Behav 1998 Aug;25:517-31.
}

\section{Question}

Do individualised computer generated nutrition information and iterative feedback reduce dietary fat intake and increase fruit and vegetable intake?

\section{Design}

Randomised controlled trial.

\section{Setting \\ The Netherlands.}

\section{Participants}

Participants were recruited through newspaper and radio advertisements. 762 participants completed the baseline questionnaire. Follow up was $85 \%(\mathrm{n}=646$, mean age $44 \mathrm{y}, 82 \%$ women).

\section{Intervention}

Participants were allocated to 1 of 3 groups, which differed by the type of nutrition information they received: a computer generated feedback letter individualised to the participant's dietary behaviour for fat, fruit, and vegetable intake and a second iterative feedback letter tailored to changes in dietary behaviour made after the first feedback letter (group 1, $\mathrm{n}=215$ ); a computer generated feedback letter, which was the same as the one that group 1 participants received, without a second iterative feedback letter (group 2, $\mathrm{n}=211$ ); and a general nutrition information letter that promoted a diet low in fat and high in fruits and vegetables (group $3, \mathrm{n}=220$ ).

\section{Main outcome measures}

Self reported fat, fruit, and vegetable intake at 4 weeks after receiving the first nutrition information letter (first post-test) and 4 weeks after participants in group 1 received the iterative feedback letter (final post-test).

\section{Main results}

At the final post-test, mean fat points per day were higher in group 3 (27.5) than in both group $1(25.6, \mathrm{p}<0.001)$ and group $2(26.2, p=0.01)$, which showed that individualised computer generated feedback alone or combined with iterative feedback reduced fat intake more than a general nutrition information letter. There was no difference in fat points between groups 1 and 2. At the final post-test, mean number of servings of vegetables per day were higher in group 1 (1.20) than in group 3 (1.08, $\mathrm{p}=0.001$ ), showing that individualised computer generated feedback plus iterative feedback increased vegetable intake more than a general nutrition information letter. There were no differences in vegetable intake between groups 1 and 2 or between groups 2 and 3 . At the final post-test, mean number of servings of fruit per day were higher in group 1 (2.45) than in group 3 (2.02, $\mathrm{p}=0.006)$, again showing the benefit of adding iterative feedback to individualised computer generated feedback. There were no differences in fruit intake between groups 1 and 2 or between groups 2 and 3 .

\section{Conclusion}

Computer generated individualised feedback plus iterative feedback had a greater effect on reducing fat and increasing fruit and vegetable intake than general nutrition information.

Source of funding: The Dutch Cancer Society.

For correspondence: Dr J Brug, Department of Social Studies, Open University, PO Box 2960, 6401 DL Heerlen, the Netherlands. Fax +31 455762939 .

\section{Commentary}

Individualised computer generated information and iterative feedback have been shown to successfully change health related behaviour. ${ }^{1}$ This model was developed with the belief that a more permanent change can be achieved if an intervention is matched to the individual's stage of change. In this study, individualised plus iterative feedback were used to tailor the intervention to the individual's "stage." In other dietary intervention studies, positive change occurred over the first 2-3 months, but long term change was more difficult to achieve without regular prompting. ${ }^{2}$ The results of this study must be interpreted with caution because the follow up period was short (10 wks).

Study participants were mainly young, educated women. Participants were not overweight and therefore the need for dietary change was small. Similar changes may not be achievable in an overweight population. The magnitude of change, although statistically significant, may not be large enough to affect morbidity and mortality.

This study has relevance to community nurses, general practice nurses, and health promoters. It shows that a personalised intervention works, emphasises the need to heighten awareness of acceptable dietary intake, and motivates change of undesirable eating habits. Dietary feedback should be relevant to the individual and should include specific aims so that guidance is clear and practical. Iterative dietary feedback in addition to the initial individualised information is effective in increasing the longer term impact. These techniques have always been thought to be impractical because of the costs of tailoring interventions to individuals. Computer technology, which was used in this study, may help make this approach more cost effective and potentially useful in community settings.

Susan E Campbell, RN, MSc Trial Coordinator Health Services Research Unit University of Aberdeen Aberdeen, $U K$

1 Sutton S. Transtheoretical model of behaviour change. In: Baum A, Newman S, Weinman J, et al, editors. Cambridge handbook of psychology, health, and medicine. Cambridge: Cambridge

2 Patterson RE, Kristal AR, Glanz K, et al. Am J Prew Med 1997;13:271-6. 